\title{
Solution of Helmholtz Equation Using Finite Differences Method in Wires Have Different Properties along X-Axis
}

\author{
Ayse Nihan Basmaci Filiz \\ Corlu Vocational School, Tekirdag Namik Kemal University, Tekirdag 59860, Turkey
}

\begin{abstract}
In this paper, frequencies of electromagnetic wave in a conductive wire are investigated theoretically. The conductive wire has specific variable material properties along the length of itself. Furthermore, material properties varying along the length of the wire are determined according to a specific mathematical function. In addition, the central finite difference method is applied to the Maxwell equations. The accuracy of the mode 1 frequency parameter is obtained to be $0.06 \%$. This result can be obtained by assuming the number of conductive wire nodes 20 . The obtained results show a very good agreement with the exact solution results.
\end{abstract}

Key words: Finite differences method, Helmholtz equation, Electromagnetic wave.

\section{Introduction}

Due to the fast developments in applied mathematics, vibrations, acoustics and electromagnetics, requirement of solutions for Hemholtz partial differential equation increases more and more. Depending on the developments, it is important to examine the subject of electromagnetic wave in theory. Maxwell's equations are obtained and the electromagnetic wave is examined in details [1-3]. According to Mathews and Fink's [4] study, the mathematical base of finite differences method and the use of Taylor series equation and the $\mathrm{O}\left(\mathrm{h}^{2}\right)$ truncation operations are described. In Mastryukov and Mikhailenko's [5] study, the wave characteristic of a two-dimensional structure with a total of 25 nodes arranged diagonally in each of the five pieces has been analysed using the finite difference method.

Wave characteristic of a two-dimensional structure is investigated. The two-dimensional structure has nodes which are aligned in a linear row on the $x$ and $y$ axes. [6].

Corresponding author: Ayse Nihan Basmaci Filiz, Dr., research fields: electromagnetic field and wave theory, numerical methods in electromagnetism, microwave theory, planar microwave filters.
In Acharjeea et al.'s [7] study, wave behaviour is examined using the finite differences time domain method (FDTD). In this study, the wave also reflects by hitting a specific surface. The equation of the 3-dimensional Helmholtz is solved by using the iterative methods [8].

Tinga [9] has studied on a wave behaviour of a specific structure that has non-uniform material properties. In the study of Tinga, variation of the frequencies depending on non-uniform material properties of the used structure has been examined.

In this article, material properties varying along the length of a conductive wire are determined according to the exponential and linear mathematical functions. Frequencies of electromagnetic wave in the conductive wire are obtained.

\section{Theoretical Analysis}

Firstly, a conductive wire is divided into $n$ nodes as shown in Fig. 1. Material properties of the wire vary along one end to another.

The distance between each node is called number of steps, $h$. As can be seen, $r(x)$ is defined as a specific function.

By using backward Eq. (2) and forward Eq. (1) taylor series expansion: 


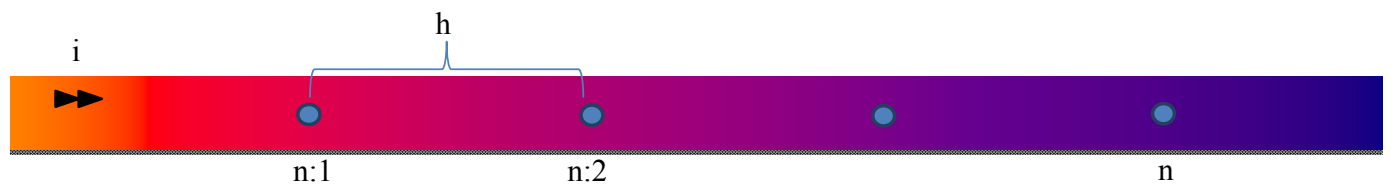

Fig. 1 Central finite differences node points.

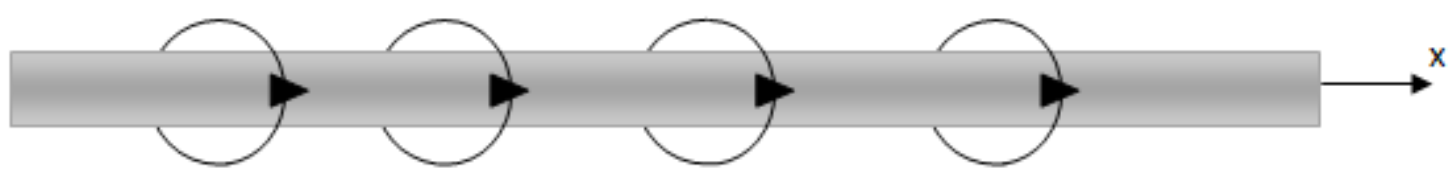

Fig. 2 Homogenous conductive wire.

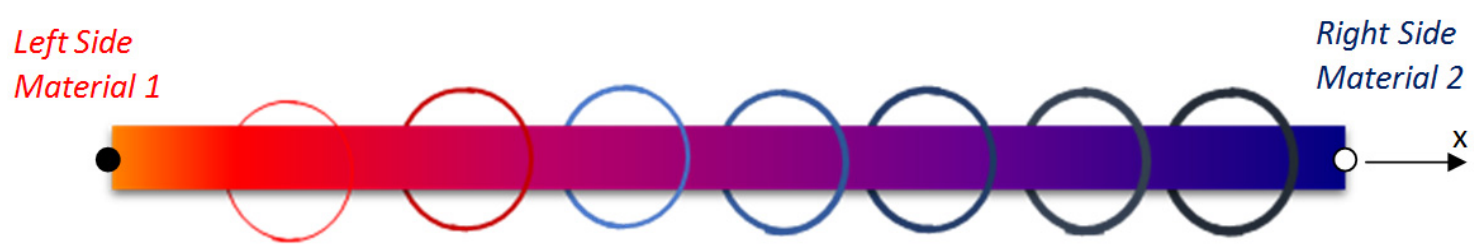

Fig. 3 Nonhomogeneous conductive wire.

$$
\begin{gathered}
r(x+\mathrm{h})=r(x)+\frac{\mathrm{h}}{1 !} \frac{\partial r}{\partial x}+\frac{\mathrm{h}^{2}}{2 !} \frac{\partial^{2} r}{\partial x^{2}}+\frac{\mathrm{h}^{3}}{3 !} \frac{\partial^{3} r}{\partial x^{3}}+ \\
\frac{\mathrm{h}^{4}}{4 !} \frac{\partial^{4} r}{\partial x^{4}}+\cdots \\
r(x-\mathrm{h})=r(x)-\frac{\mathrm{h}}{1 !} \frac{\partial r}{\partial x}+\frac{\mathrm{h}^{2}}{2 !} \frac{\partial^{2} r}{\partial x^{2}}-\frac{\mathrm{h}^{3}}{3 !} \frac{\partial^{3} r}{\partial x^{3}}+ \\
\frac{\mathrm{h}^{4}}{4 !} \frac{\partial^{4} r}{\partial x^{4}}+\cdots
\end{gathered}
$$

Substraction of Eqs. (1) and (2) is divided into 2 in order to find central differences first derivative expression in $\mathrm{O}\left(\mathrm{h}^{2}\right)$ order, Eq. (4):

$$
\begin{gathered}
r(x+\mathrm{h})-r(x-h)=2 \mathrm{~h} \frac{\partial r}{\partial x}+\frac{\mathrm{h}^{3}}{3} \frac{\partial^{3} r}{\partial x^{3}} \\
\frac{\partial r}{\partial x}=\frac{r(x+\mathrm{h})-r(x-\mathrm{h})}{2 \mathrm{~h}}
\end{gathered}
$$

Sum of Eqs. (1) and (2) is given in Eq. (5):

$$
r(x+\mathrm{h})+r(x-h)=2 r(x)+\mathrm{h}^{2} \frac{\partial^{2} r}{\partial x^{2}}
$$

In order to express Eq. (6), Eq. (5) is simplified and a second derivative expression is obtained [4]:

$$
\frac{\partial^{2} r}{\partial x^{2}}=\frac{r(x+\mathrm{h})-2 r(x)+r(x-h)}{h^{2}}
$$

In a source free, linear isotropic and homogenous region, Maxwell's equations are [3]:

$$
\begin{aligned}
& \nabla \times \bar{E}=-j \omega \mu \bar{H} \\
& \nabla \times \bar{H}=-j \omega \epsilon \bar{E}
\end{aligned}
$$

It should be noted that, $\mu$ is permeabilty, $\epsilon$ is permittivity, $\bar{E}$ is electrical field and $\bar{H}$ is magnetic field.

Eq. (8) is obtained by using Eqs. (7a) and (7b):

$$
\frac{\partial^{2} H}{\partial x^{2}}+\omega^{2} \mu \epsilon H=0
$$

In Eq. (8), $k$ indicates wave number in a homogenous wire as shown in Fig. 2.

The nonhomogenous conductive wire is given in Fig. 3. It has different material properties along the length of itself and the wire length, $L$ is defined as $1 \mathrm{~m}$.

Eq. (9a) is obtained due to the change in the material properties of the nonhomogenous wire:

$$
\frac{\partial^{2} H}{\partial x^{2}}+k r(\omega)^{2} H=0
$$

$k r(\omega)$ is the material property characteristics function of the nonhomogenous wire including the frequencies of the wire.

$$
k r=\sqrt{\mu \epsilon} \omega
$$

When material property characteristic function of the nonhomogeneous wire $(k r)$ is expressed in exponential form, Eq. (10) is obtained where $\gamma$ is 
indicated as an exponential constant. It should be noted that, linear results are obtained in the high values of $\gamma$.

$$
k r=\left(\frac{k 2-k 1}{e^{\gamma+1}}\right) e^{\gamma x}+\left(k 1+\frac{k 1-k 2}{e^{\gamma+1}}\right)
$$

According to the $k r$ function in Eq. (10), Eq. (11) is obtained.

The effect of the exponential constant, $\gamma$ on the $k r$ function can be clearly seen from Fig. 4. It should be noted that, the left side of the given graph in Fig. 4 is labelled as $k 1$ whereas right side is labelled as $k 2$.

Quadrative derivative expression is applied to

$$
\left[\begin{array}{cc}
\left(k r(\omega)^{2}-\frac{2}{h^{2}}\right) & \frac{1}{h^{2}} \\
\frac{1}{h^{2}} & \left(k r(\omega)^{2}-\frac{2}{h^{2}}\right) \\
0 & \frac{1}{h^{2}}
\end{array}\right.
$$

Helmholtz equation. In this purpose, Eq. (6) and Eq. (10) are replaced to Eq. (9a):

$$
\frac{r(x+h)-2 r(x)+r(x-h)}{h^{2}}+k r^{2} r(x)=0
$$

Eq. 11 is obtained, thus. Eq. 11 is also expressed in its indices notation form as follows:

$$
\frac{r_{n+1}+r_{n-1}}{h^{2}}+\left(k r^{2}-\frac{2}{h^{2}}\right) r_{n}=0
$$

If node number, $n$ is defined as 3 , the solution is obtained as Eq. (13a). If the node number, $n$ is increased, the matrix expands diagonally.

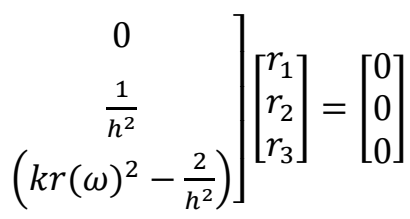

In Eq. (13a), if determinant solution of $3 \times 3$ matrix is solved as equals to zero, nontrivial solution is obtained (Eq. (13b)).

$$
\left|\begin{array}{ccc}
\left(k r(\omega)^{2}-\frac{2}{h^{2}}\right) & \frac{1}{h^{2}} & 0 \\
\frac{1}{h^{2}} & \left(k r(\omega)^{2}-\frac{2}{h^{2}}\right) & \frac{1}{h^{2}} \\
0 & \frac{1}{h^{2}} & \left(k r(\omega)^{2}-\frac{2}{h^{2}}\right)
\end{array}\right|=0
$$

To solve the Eq. (13b), the determinant of the coefficient matrix is equalized to zero. Accordingly, the $\omega$ values are obtained as the frequency parameters.

\section{Results and Discussions}

Fig. 5 shows that, the accuracy of the resulting $\omega$

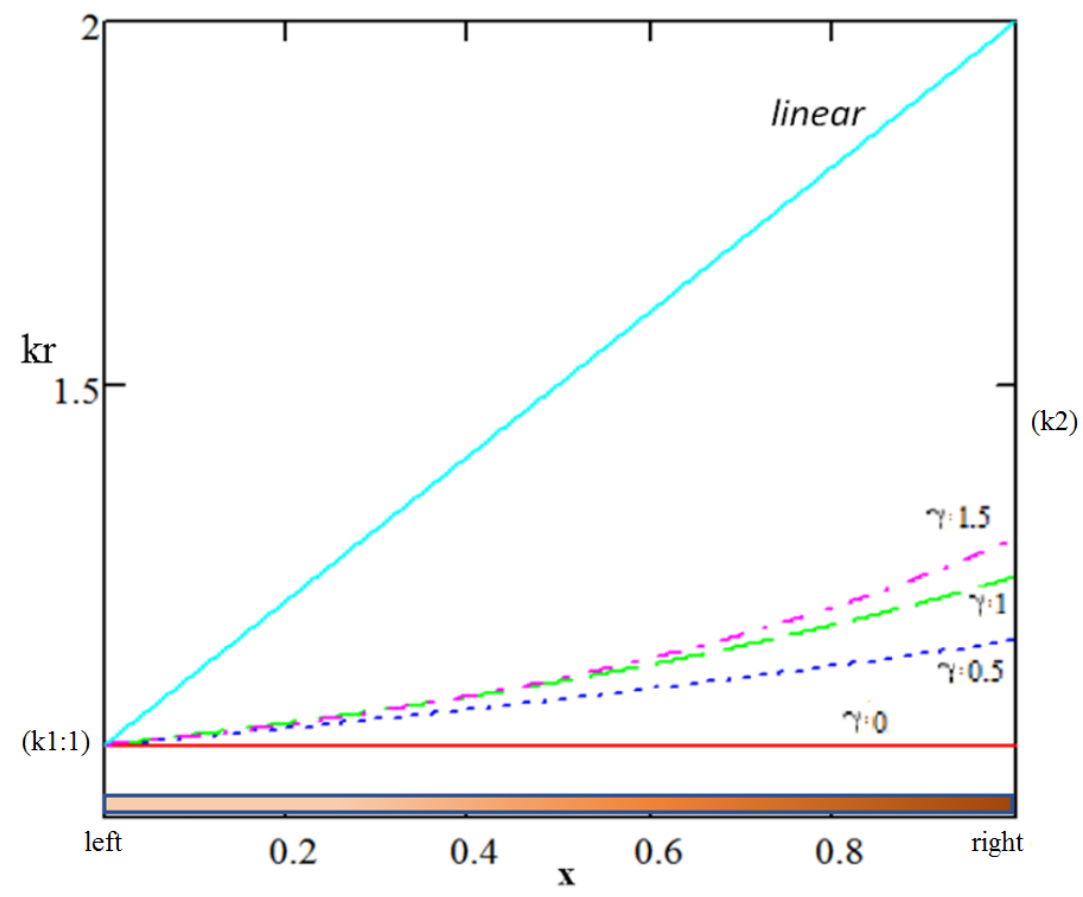

Fig. 4 Adjustment of variable material property characteristic due to exponential constant. 
Solution of Helmholtz Equation Using Finite Differences Method in

Wires Have Different Properties along X-Axis

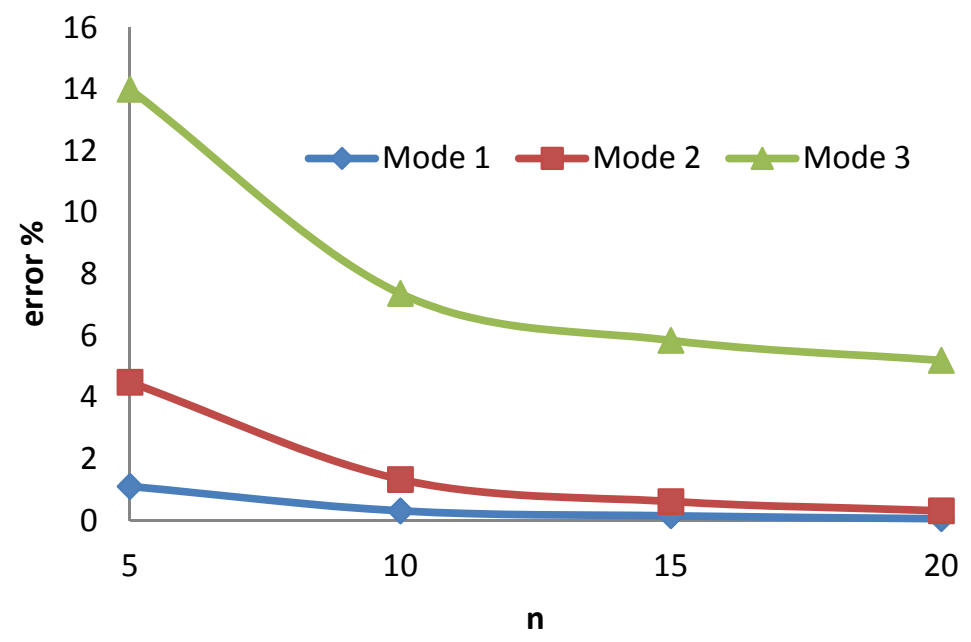

Fig. 5 Convergence study of finite differences method.

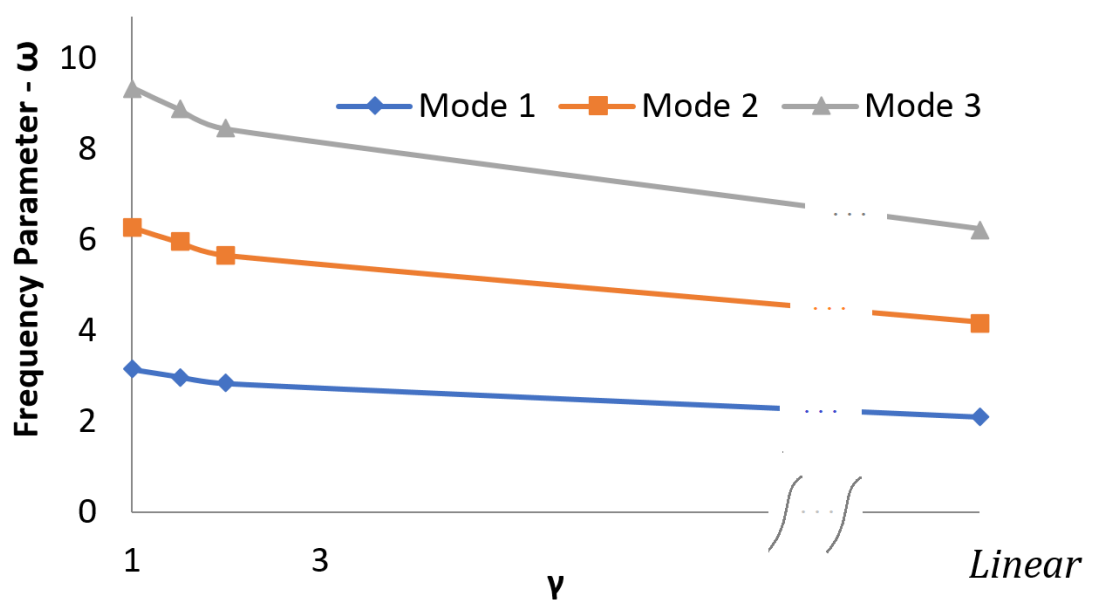

Fig. 6 Variation of frequency parameters due to $\gamma(k 2 / k 1: 2 / 1)$.

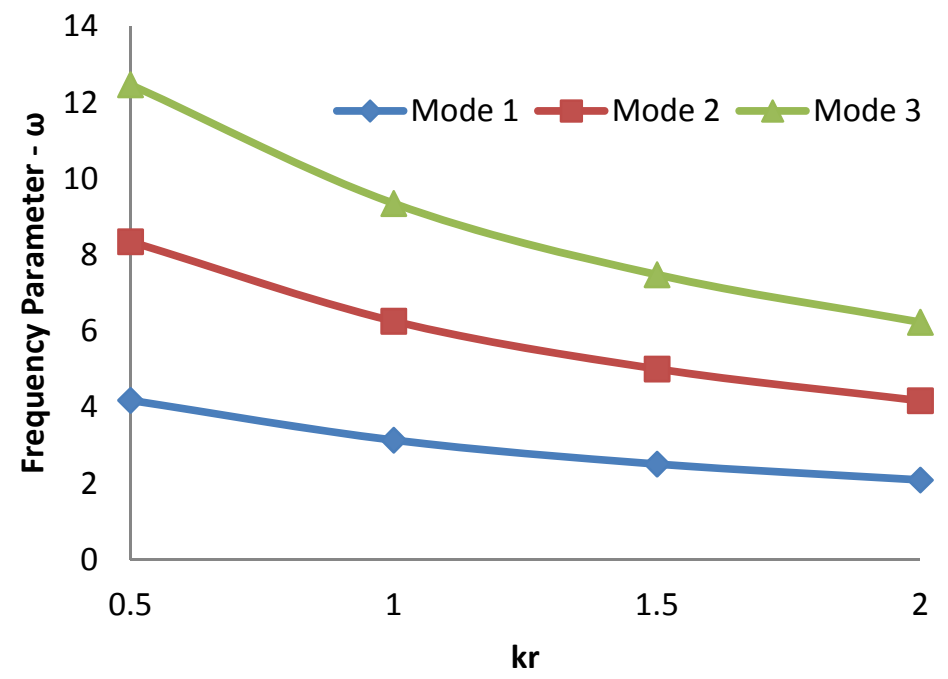

Fig. 7 Variation of frequency parameter due to $k r$ (linear formation). 


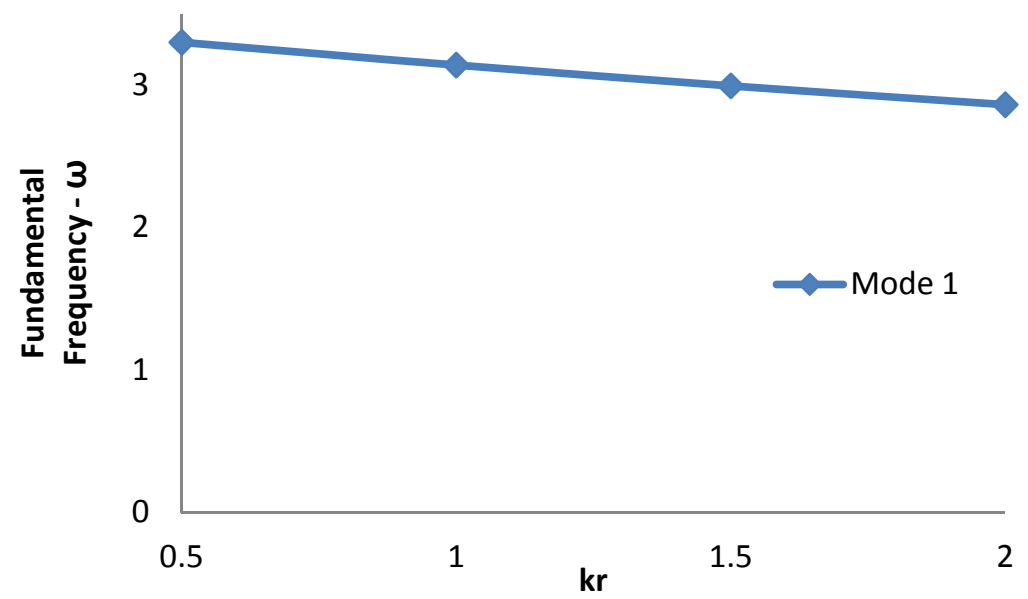

Fig. 8 Variation of fundamental frequency due to $k r$ (exponential formation).

values increases in proportion to the increase in the number of nodes. In this article, node number, $n$ is defined as 20 . It should be noted that, the fundamental frequency error rate is obtained as $0.06 \%$ compared to the exact solution. The more the modes increase, the more the frequency error rate increases. Accordingly, fundamental frequency has the smallest error rate.

In Fig. 6, as the exponential constant increases, the decrease in the dimensional frequency parameters occurs. The material property in high values of the $\gamma$ constant indicates linear behaviour. The frequencies obtained in the linear material property behaviour have the smallest values.

In Figs. 7 and 8, the effect of material property characteristic, $k r$ which is expressed in linear and exponential form, is examined on the frequency parameters. Depending on the increase in material property characteristic, $k r$, the obtained frequency values decrease as can be clearly seen from the figures.

\section{Conclusion}

In this article, effects of variable material property characteristic on electromagnetic wave frequencies were investigated. For this purpose, the frequency values of electromagnetic wave occurring in a conductive wire whose material properties changes from one end to another are obtained. Central finite difference method was utilized in obtaining electromagnetic wave frequencies. When the central finite difference method is applied, the number of nodes specified must be sufficient to reach the frequency values close to the exact frequency values. In this regard, the number of nodes for the finite difference method was defined as 20 . Obtained results show that, frequency values decrease directly proportional to the increase in the material property characteristic, $k r$.

As a future research, this analysis can also be performed for wires whose material properties changes transversely and whose cross-section area alternating along the $\mathrm{x}$-axis.

\section{References}

[1] Jordan, E. C., and Balmain K. G. 1968. Electromagnetic Waves and Radiating Systems. Englawood Cliffs, New Jersey: Prentice Hall.

[2] Kong, J. A. 1990. Electromagnetic Wave Theory (Chapter 1). New York: Wiley Interscience.

[3] Pozar, D. M. 2012. Microwave Engineering (4th ed.). London: John Wiley \& Sons.

[4] Mathews, J. H., and Fink, K. F. 2004. Numerical Methods Using Matlab (4th ed.). New Jersey, USA: Prentice-Hall Pub. Inc.

[5] Mastryukov, A. F., and Mikhailenko, B. G. 2015. "Optimal Difference Schemes for Maxwell's Equations in Solving Forward Problems of Electromagnetic Soundings." Russian Geology and Geophysics 56: 1349-56.

[6] Rylander, T., Ingelström, P., and Bondeson, A. 2013. Texts in Applied Mathematics, Computational Electromagnetics: The Finite-Differences Time-Domain 
Method. Springer.

[7] Acharjeea, D., Hasan, N., and Maity, S. 2016. "Finite Difference Time Domain simulation of Active Cancellation of Radar Echoes." Procedia Computer Science Procedia Computer Science 92: 233-6.

[8] Erlangga, Y. A. 2008. "Advances in Iterative Methods and Preconditioners for the Helmholtz Equation." Arch.
Comput. Methods Eng. 15 (1): 37-66.

[9] Tinga, W. R. 1992. "Mixture Laws and Microwave-material Interactions." In Progress in Electromagnetic Research (PIER) Monograph Series Vol. 6 on Dielectric Properties of Heterogeneous Materials, edited by Priou, A., chapter 1. New York: Elsevier, 1-40. 